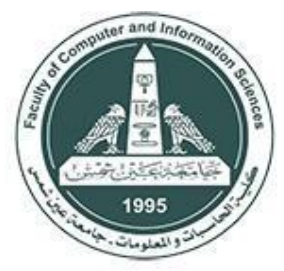

International Journal of Intelligent Computing and Information Sciences

https://ijicis.journals.ekb.eg/

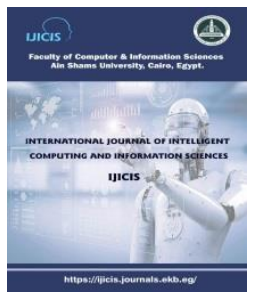

\title{
AUGMENTED REALITY SYSTEM IN TOTAL HIP ARTHROPLASTY USING TRANSVERSE ACETABULAR LIGAMENT
}

Amal Yassin*

Computer Science Department, Faculty of Computer and Information Sciences, Ain Shams University Cairo, 11566, Egypt

amal.hussein.yassin@cis.asu.edu.eg
Mamdouh Hefny

Orthopaedics Department, Warwick Hospital

Warwick, CV34 5BW, United Kingdom

mamdouh.hefny@swft.nhs.uk
Taha Elarif

Computer Science Department, Faculty of Computer and Information Sciences, Ain Shams University Cairo, 11566, Egypt taha_elarif@cis.asu.edu.eg

Received 2020-09-15; Revised 2020-11-18; Accepted 2020-12-05

\begin{abstract}
Augmented reality $(A R)$ is an interactive technology that enhances the real-world environment and its real objects by augmenting new computer-generated objects. It has already invaded various fields, and particularly medicine. AR has become an essential entity of computer-assisted surgeries (CAS), as it has already shown great results in both conventional surgeries as well as minimally invasive surgeries (MIS). It has been used intraoperatively in many surgeries, especially in total hip arthroplasty (THA). Most of the systems using AR intraoperatively in THA require pre-operative CT-scan and/or intraoperative X-ray imaging, which increases radiation exposure for both the medical staff and the patient and increases surgery time and cost. This paper aims to propose a new AR system that uses the transverse acetabular ligament (TAL) as a guide for defining the accurate position for placing the acetabulum cup in THA. In opposite to other AR systems, this system is an image-free system, which means that it does not require a pre-operative CT-scan or intraoperative $X$-ray images, thus reducing radiation exposure and the surgery time and cost.
\end{abstract}

Keywords: Augmented reality, minimally invasive surgery, orthopedics, total hip arthroplasty, transverse acetabular ligament, cup placement.

\section{INTRODUCTION}

Augmented reality (AR) is an interactive technology that enhances the real-world environment and its real objects by augmenting new computer-generated objects. It has been applied in several fields, especially in medicine. AR has become an essential entity of computer-assisted surgeries (CAS), as it has already shown great results in both conventional surgeries as well as minimally invasive surgeries (MIS). It has been used intraoperatively in many surgeries, especially in total hip arthroplasty (THA). * Corresponding author: Amal Yassin Computer Science Department, Faculty of Computer and Information Sciences, Ain Shams University, Cairo, 11566, Egypt E-mail address: amal.hussein.yassin@cis.asu.edu.eg 
Most of the systems using AR intraoperatively in THA require pre-operative CT-scan and/or intraoperative X-ray imaging for placing the acetabular cup in the correct position. As a result, it increases radiation exposure for both the medical staff and the patient and increases surgery time and cost.

In THA, the accurate positioning of the acetabular cup requires a three-dimensional alignment in the axial, coronal, and sagittal planes. It is essential for achieving an adequate postoperative range of motion. Since the accurate acetabular orientation is still controversial, the acetabular cup mal-alignment is the most common error in THA [1].

Yassin et al. [2] made a comparison between two systems that use AR in THA. The first system in [3] was used as a navigation tool, in which AR was used intraoperatively to let the surgeon plan the position of the cup using two C-arm X-ray images acquired intraoperatively from two different perspectives and then align the real impactor on the monitor with the virtual planned impactor in all of the perspectives provided to place the real acetabular cup in the planned position correctly. The system showed accurate results in translation, abduction, and anteversion angles. The second system [4] was used as a measurement tool, in which AR was used to measure the abduction and anteversion angles of the acetabular cup. A preoperative CT-scan was required for planning the position of the cup, then THA was performed using the conventional mechanical guide. The image of the virtual cup and impactor was superimposed on the view of the real cup and impactor, then the image can be managed through the abduction and anteversion buttons on a touch panel. The system showed accurate results in anteversion angles. Although both systems provided better results than those of conventional surgery, they required preoperative CT-scan or intraoperative X-ray imaging for planning the accurate position of placing the acetabular cup which increases radiation exposure for both the medical staff and the patient.

As a result, we propose a new system that uses AR to define the accurate angle for positioning the acetabular cup without using preoperative CT-scan or intraoperative X-ray imaging which reduces radiation exposure and surgery time and cost. The system uses the transverse acetabular ligament (TAL) as a guide. The TAL, as shown in Figure 1, is a portion of the acetabular labrum forming a fibrocartilaginous rim that surrounds the bony acetabulum and straddles its inferior limit. It consists of strong, load bearing, flattened fibres, which form a bridge that crosses the inferior acetabular notch and converts it into a foramen through which the nutrient vessels enter the joint. In the normal hip, the TAL provides, in association with the labrum, part of the load-bearing surface for the femoral head [5].

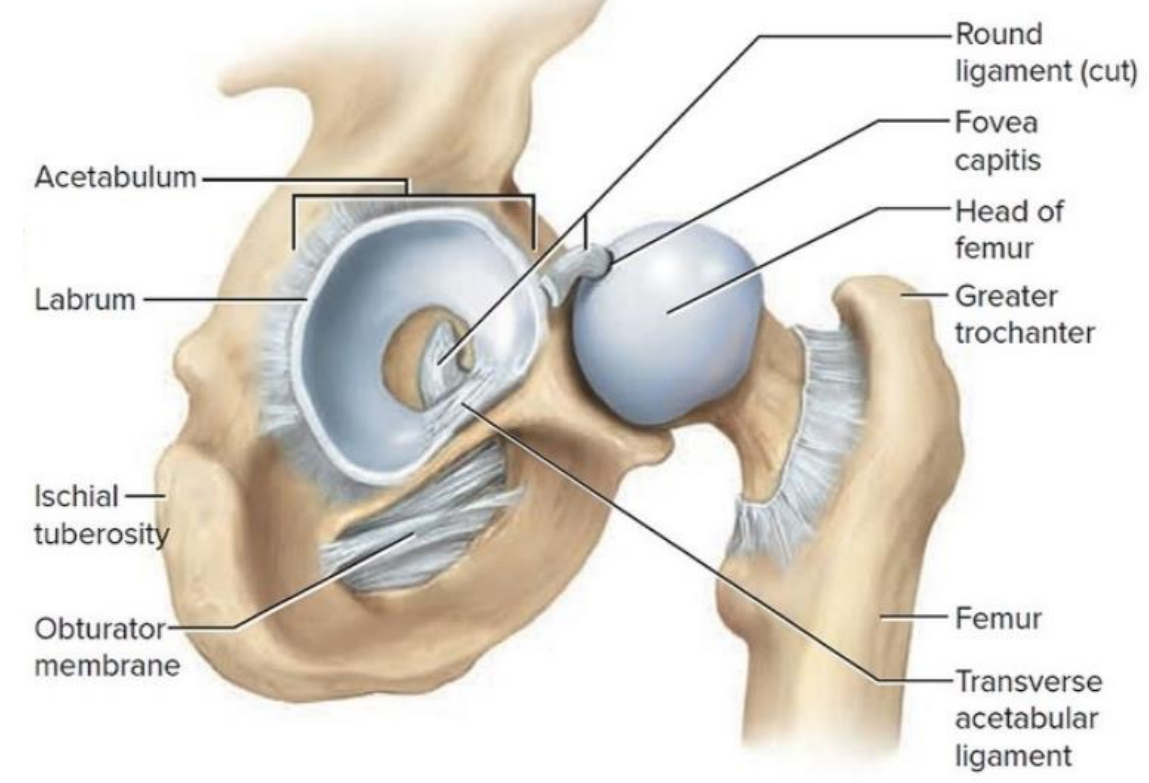

Figure 1: Lateral view showing the TAL after retracting the femur 
For ages, the surgeons performing THAs could not figure out the function of the TAL and some of them considered it as an obstacle in placing the cup until it was used as an intraoperative landmark by Archbold et al. [6] to control the positioning of the acetabular cup. They made a clinical study on 1000 THAs in which the TAL was used as a landmark to reliably obtain anteversion, height, and depth close to those of the natural hip. The TAL was successfully identified intraoperatively in $99.7 \%$ of the cases, with only $0.6 \%$ dislocated cases over an 8 to 41 -month follow-up period.

Many studies were performed to compare the conventional free-handed cup placement and the cup placement using the TAL as a guide [7]. It was demonstrated that the latter provides more accurate anteversion, as it provides lower variability in cup anteversion. It helped reduce the primary dislocation rate from $3.7 \%$ to $1 \%$ in [5], and $0.6 \%$ in [8]. Also, Meermans et al. [9] showed that postoperative radiographs assessments demonstrated that no acetabular cups fixed using the TAL as a guide fell outside the Lewinnek safe zone, compared with $20 \%$ of free-handed acetabular cups that did.

Using the TAL as a guide has many advantages over the conventional method:

1. The TAL can be identified in most cases.

2. It is a specific reference for each patient and is independent of patient positioning.

3. No additional instrument/imaging is required to estimate the anteversion angle.

4. It helps restore the acetabular joint centre if the cup is cradled by the TAL.

5. It shows an acceptable dislocation rate with the posterior approach.

6. The technique is simple and very useful especially in MIS.

It was known that the TAL does not help with inclination, but the authors in [10] recommended $35^{\circ}$ of apparent operative inclination (AOI) for the surgeons using the posterior approach to adjust the inclination. While the authors in [11] \& [12] demonstrated that the cup inclination was shown to be acceptable in the cases where the acetabular cup position was flush or within $5 \mathrm{~mm}$ proximal to the TAL as shown in

Figure 2. The only point to keep in mind while using the TAL for controlling version in hips with OA secondary to dysplasia that it is not a reliable guide [13], although the authors in [10] use it as a guide as they believe that the TAL and labrum reimburse any abnormalities in the bones.

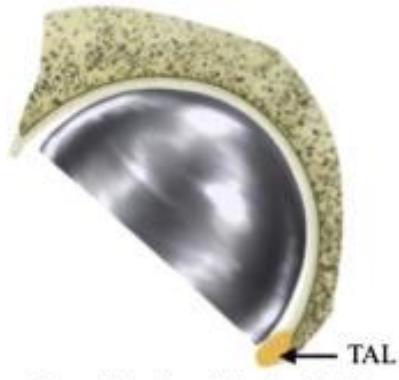

Cup flush with the TAL $(0 \mathrm{~mm})$

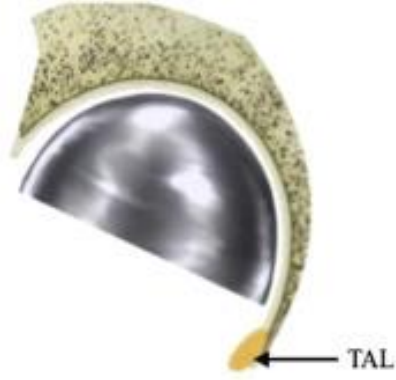

Cup $5 \mathrm{~mm}$ proximal from the TAL $(-5 \mathrm{~mm})$

Figure 2: Different positions of the cup leading to acceptable cup inclination

[11]

\section{METHODOLOGY}

The proposed system is to be used in THA to guide surgeons intraoperatively. The system uses the TAL as a guide to define the accurate angle for fixing the cup inside the acetabulum without the need for a preoperative CT-scan or intraoperative X-ray images. The system only requires the existence of a smartphone that is placed inside a sterile sealable transparent sheath, and the application, developed by the authors, has to be pre-installed on that smartphone. The proposed system increases the accuracy of placing the acetabular cup while reducing radiation exposure and surgery time and cost. 
The accurate position of the acetabulum component can be achieved by controlling both the (1) anteversion and (2) inclination of the cup. Firstly, to control (1) the anteversion of the cup, the face of the cup has to be placed parallel to the TAL [10] as shown in Figure 3. The ability of the surgeon to perceive the straightness and parallelism of the face of the cup and the TAL seems to be a trivial and precise judgment. However, von Helmholtz [14] made observations using his distorted chessboard figure to demonstrate that this judgment is not always correct [15]. As a result, our proposed system provides accurate results regarding the parallelism of the face of the cup and the TAL to control the anteversion. Another advantage of the system is that it does not require drawing a line parallel to the TAL on the drape that covers the skin adjacent to the wound of the patient in the cases that the TAL is not visible. Finally, To control (2) the inclination of the cup, the surgeon has two options; to use a digital inclinometer to achieve $35^{\circ}$ of AOI as shown in [10], or to follow the aspect of the authors in [11] \& [12] and adjust the cup position to be flush with the TAL.
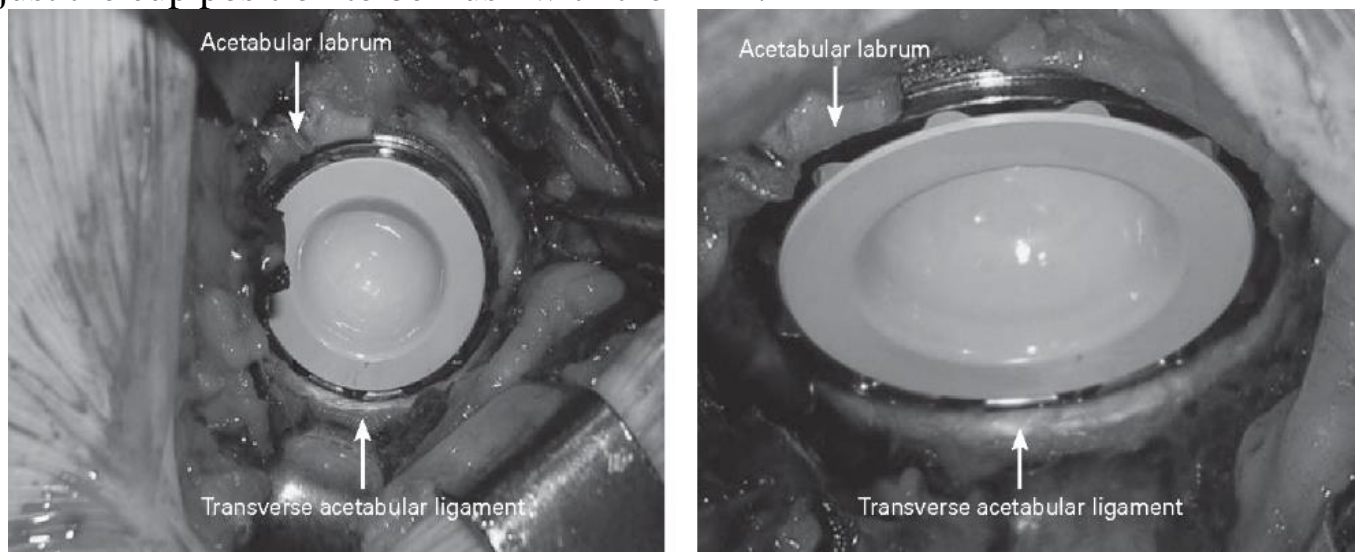

Figure 3: Accurate position of the acetabular cup using the TAL technique [6]

\subsection{Surgical Techniques}

The surgeon can follow the next steps to use the system:

1. The surgeon dislocates the femoral head.

2. Using the pins, anti-retractor, and inferior tear-drop retractor, the surgeon can follow the technique of Beverland in [10] to identify the TAL according to the grade of the TAL and then ream the acetabulum labrum as in Figure 4.

3. The surgeon determines the size of the acetabular cup depending on the size of the reamer.

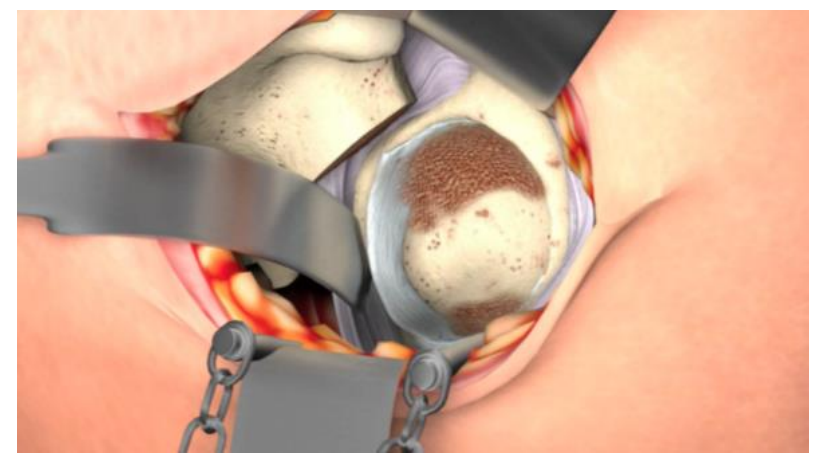

Figure 4: A $360^{\circ}$ fixed-window exposure of the acetabulum showing the TAL and the acetabulum labrum prepared for placing the acetabulum cup [16] 
4. The surgeon touches the screen to render the $3 \mathrm{D}$ model of the TAL in the centre of the screen as shown in Figure 5. The model is first shown as a green rod.

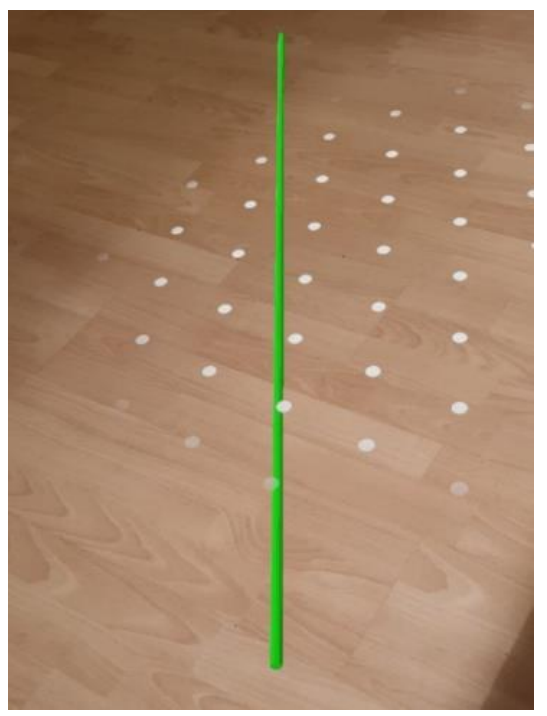

Figure 5: The 3D model of the TAL is shown on the screen

5. The system detects the cup whenever it is shown on the screen. Then, the absolute difference between the angle of the TAL model and the angle of the face of the cup is shown on the screen as shown in Figure 6.

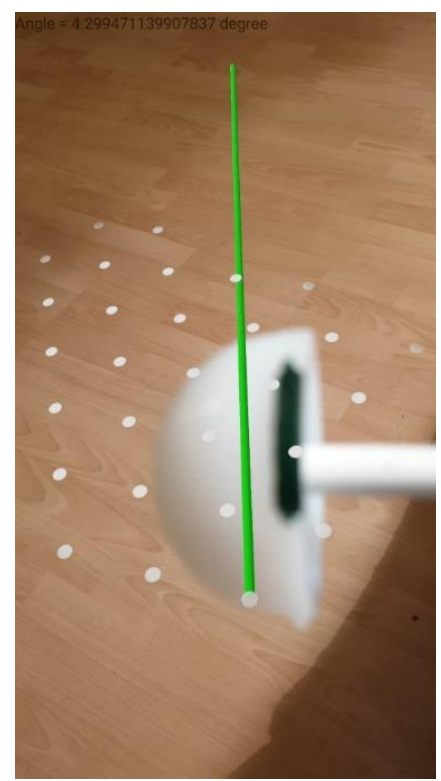

Figure 6: Detecting the cup and showing the angle it makes with the 3D model

6. The surgeon places the cup inside the acetabulum as shown in Figure 7. 


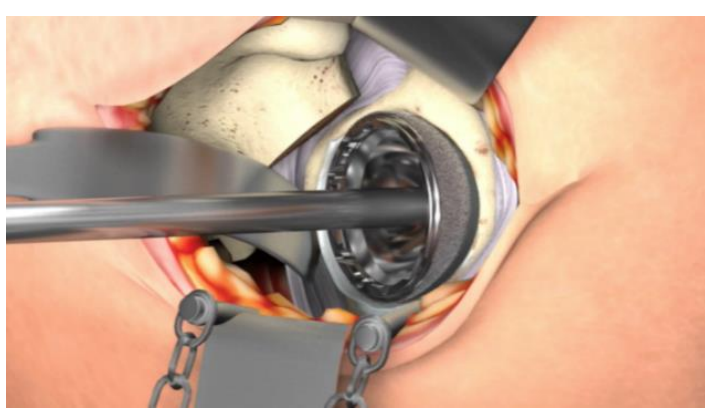

Figure 7: The cup is placed inside the acetabulum [16]

7. The surgeon adjusts the face of the cup until it becomes parallel to the TAL model, then the colour of the TAL model is changed into blue. The system allows a divergence of $1^{\circ}$ in the anteversion angle as shown in Figure 8.
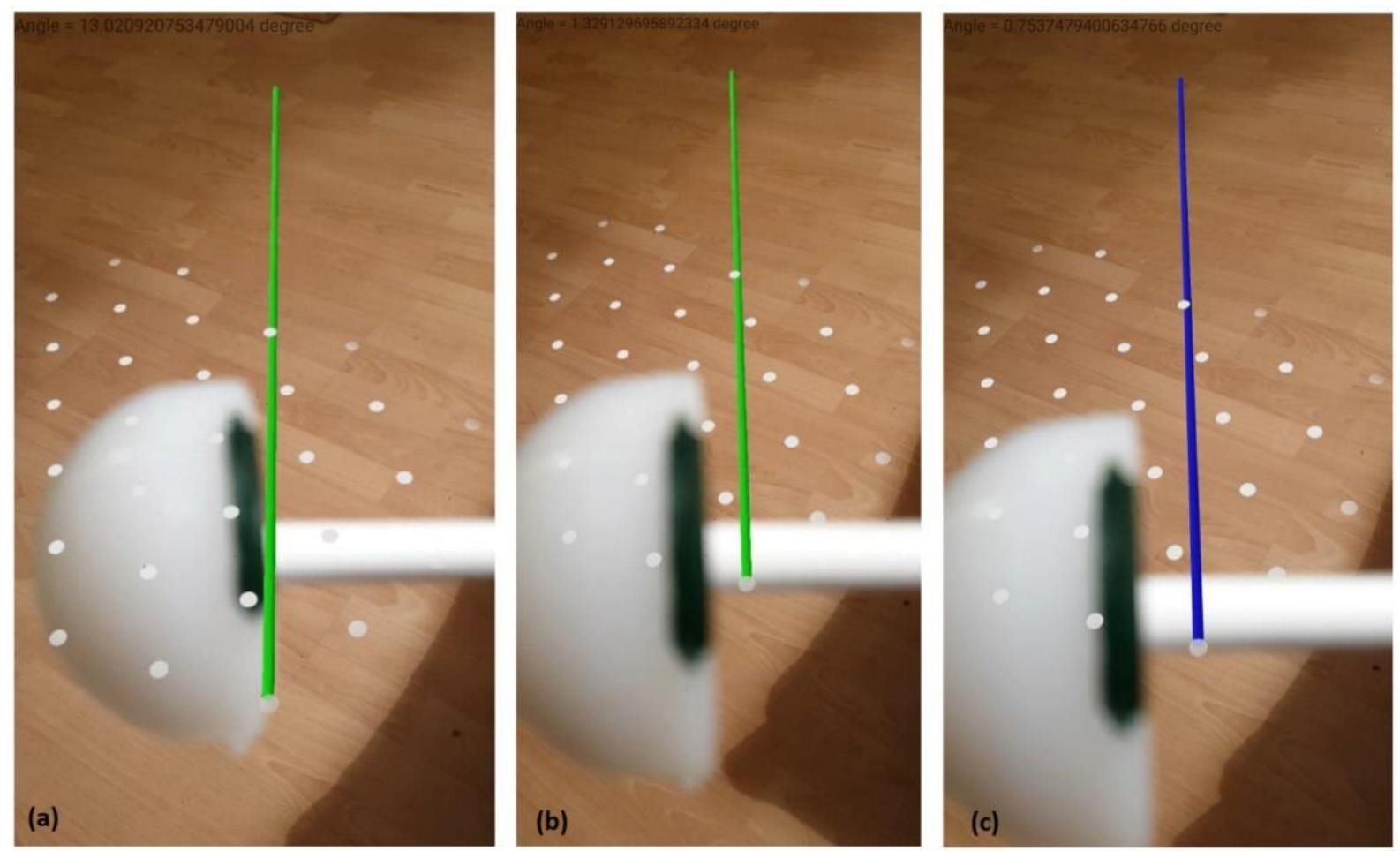

Figure 8: (a) The face of the cup is not parallel to the TAL model (angle $=\mathbf{1 3 . 0 2}^{\circ}$, model colour is green) - (b) The face of the cup is not parallel to the TAL model (angle $=1.3^{\circ}$, model colour is green) - (c) The face of the cup is parallel to the TAL model (angle $=\mathbf{0 . 7 5}^{\circ}$, model colour is blue)

8. After adjusting the anteversion of the cup, the surgeon elevates the cup to an angle of $35^{\circ}$ adjust the inclination of the cup as shown in Figure 9. 


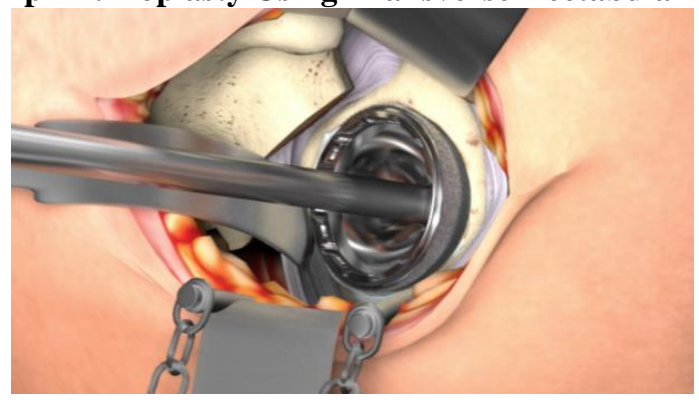

Figure 9: Adjusting the cup inclination by elevating the cup to an angle of $35^{\circ}$ [16]

9. Finally, the surgeon impacts the cup and then inserts the liner as shown in Figure 10 and Figure 11 respectively.

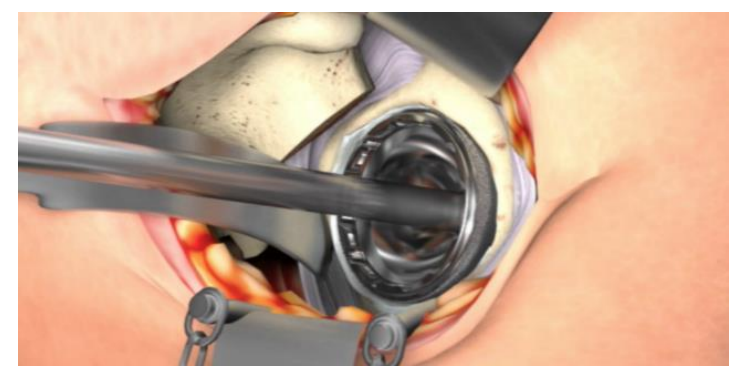

Figure 10: Impacting the cup [16]

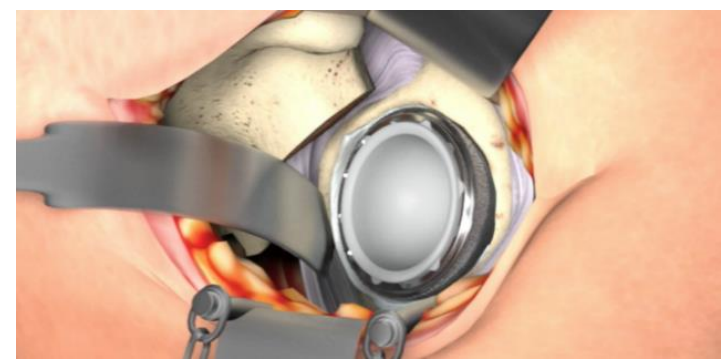

Figure 11: Inserting the liner of the cup [16]

\subsection{System Methodology}

In our system, we use Android Studio as the development application, with which ARCore and OpenCV are integrated. ARCore is the platform of Google that is used for building AR experiences by providing many software development kits (SDKs). These SDKs provide native application programming interfaces (APIs) for all the necessary AR features such as motion tracking, environmental understanding, and light estimation. These APIs make it easier for the developers to build new AR experiences or enhance existing applications with AR features. In our system, we use Sceneform SDK, one of the SDKs that ARCore provides. It is simple and straightforward to use to render realistic 3D scenes without having to learn OpenGL. It includes:

- A high-level scene graph API.

- A realistic physically-based renderer provided by Filament.

- An Android Studio plugin for importing, viewing, and building 3D assets.

Then, we used OpenCV to detect the TAL model and the face of the cup using their colours.

The system methodology comprises the following steps as shown in Figure 12. 
1. Sceneform SDK Tasks:

a. Detecting the surfaces of the scene.

b. Importing and viewing the 3D asset of the TAL model.

c. Updating the scene with all anchors and models in the correct places and with the planned colours.

2. OpenCV Tasks:

a. Detecting the model and the cup by the colour.

1. Apply Gaussian blur filter to the frame.

2. Change the frame into the HSV colour range.

3. Detect only blue and green objects.

4. Find the contour of each object.

5. Find the rotated rectangle of each object.

6. Find the angle of each object.

b. Finding the two largest contour areas, the contour areas of the TAL model and the cup, and their corresponding angles.

c. Calculating the absolute difference between the two angles, then show it on the screen.

d. Updating the colour of the TAL model according to the absolute difference between the two angles, blue if the absolute difference is less than or equal to 1 , and green otherwise.

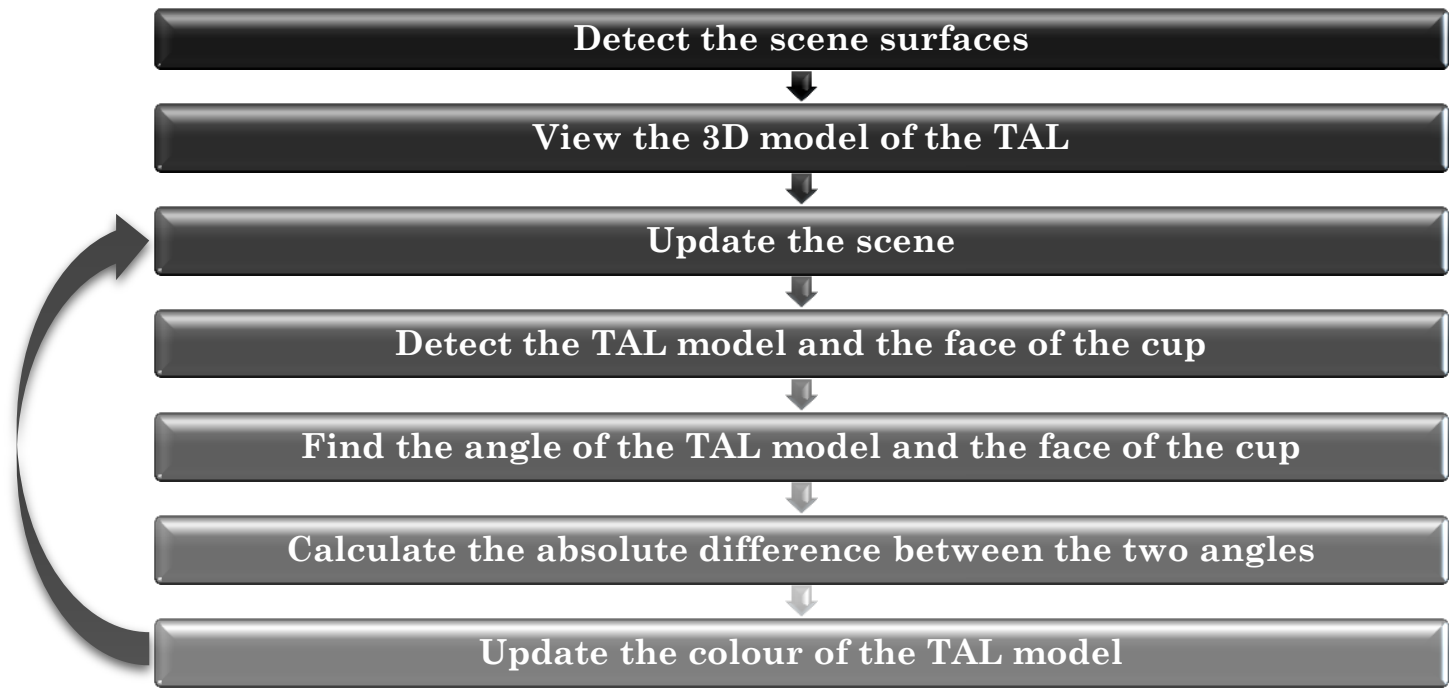

Figure 12: System Methodology

\section{DISCUSSION}

We proposed a system that uses AR intraoperatively in THA to guide the surgeon in placing the acetabulum component in an accurate position. The system used the TAL as a guide, which is now used as a landmark to reliably obtain anteversion, height, and depth close to those of the natural hip.

The human eye has a margin for error in identifying parallel lines. In Figure 8 (b), although the cup placement may seem to be parallel to the green line, there was $1.3^{\circ}$ divergence. The use of augmented reality systems may improve the alignment of the cup placement. Furthermore, less experienced surgeon may find this tool useful during their learning curve.

The proposed system used Android Studio, ARCore, and OpenCV to get satisfying results for both AR and Computer Vision. The ARCore provides Sceneform SDK which is, in our system, responsible for detecting the surfaces of the scene, importing and viewing the 3D asset of the TAL model, and finally 

updating the scene with all anchors and models in the correct places and with the planned colours. OpenCV was responsible for detecting the model and the cup using their colours, finding their angles, calculate the absolute difference between the angles, and finally update the colour of the TAL model. The limitation of our system is that we did not have an opportunity to use it in a real-world operating theatre.

\section{CHALLENGES AND FUTURE WORK}

Some challenges were identified and hence, need more future work. A new update of the system can be made to enhance the current version in the following points:

1. The colours of the markers were not accurately detected in poor illumination conditions, so better techniques may be implemented to detect the objects in different illumination conditions.

2. The 3D model is augmented onto the real world without detecting the real TAL, hence the 3D model is not accurately adjusted on the real TAL. Other techniques may be implemented to detect the real TAL and adjust the 3D model according to its position in the real world.

3. Detecting the model and the cup as objects to avoid using markers for detection.

4. Using more landmarks and anatomical structures to increase the reliability of the system.

5. Applying the system in real-life intraoperatively to verify its use.

\section{CONCLUSION}

We proposed a system that uses AR intraoperatively in THA to guide the surgeon in placing the acetabulum component in an accurate position. The system used the TAL as a guide, which is now used as a landmark to reliably obtain anteversion, height, and depth close to those of the natural hip. The system used Android Studio, ARCore, and OpenCV to get satisfying results for both AR and Computer Vision.

\section{REFERENCES}

[1] A. Viste, J. Chouteau, R. Testa, L. Chèze, M. H. Fessy, and B. Moyen, "Is transverse acetabular ligament an anatomical landmark to reliably orient the cup in primary total hip arthroplasty?," Orthopaedics and Traumatology: Surgery and Research, vol. 97, no. 3, pp. 241-245, May 2011, doi: 10.1016/j.otsr.2010.07.012.

[2] A. Yassin, T. Elarif, and M. Hefny, “Augmented Reality Systems in Total Hip Arthroplasty,” 2019, [Online]. Available: https://www.researchgate.net/publication/338402149.

[3] J. Fotouhi et al., Technical note: an augmented reality system for total hip arthroplasty, vol. 10576. SPIE, 2018.

[4] H. Ogawa, S. Hasegawa, S. Tsukada, and M. Matsubara, "A Pilot Study of Augmented Reality Technology Applied to the Acetabular Cup Placement During Total Hip Arthroplasty," J Arthroplasty, vol. 33, no. 6, pp. 1833-1837, 2018, doi: 10.1016/j.arth.2018.01.067.

[5] D. Beverland, "The Transverse Acetabular Ligament: Optimizing Version," Orthopedics, vol. 33, p. 631, Sep. 2010, doi: 10.3928/01477447-20100722-22.

[6] H. A. P. Archbold, B. Mockford, D. Molloy, J. Mcconway, L. Ogonda, and D. Beverland, "The transverse acetabular ligament: an aid to orientation of the acetabular component during primary total hip replacement A PRELIMINARY STUDY OF 1000 CASES INVESTIGATING POST-OPERATIVE STABILITY,”vol. 88, no. 7, 2006, doi: 10.1302/0301-620X.88B7.

[7] L. Zagra, "Abstracts from the 10Th Congress of the European Hip Society," HIP International, vol. 22, no. 4, pp. 405-478, May 2012, doi: 10.5301/HIP.2012.9524.

[8] A. Viste, J. Chouteau, R. Testa, L. Chèze, M. H. Fessy, and B. Moyen, "Is transverse acetabular ligament an anatomical landmark to reliably orient the cup in primary total hip arthroplasty?," Orthopaedics and Traumatology: Surgery and Research, vol. 97, no. 3, pp. 241-245, May 2011, doi: 10.1016/j.otsr.2010.07.012.

[9] M. Abolghasemian et al., "Reconstruction of massive uncontained acetabular defects using allograft with cage or ring reinforcement: An assessment of the graft's ability to restore bone stock and its impact on the outcome of rerevision," Bone and Joint Journal, vol. 96 B, no. 3. British Editorial Society of Bone and Joint Surgery, pp. 319-324, 2014, doi: 10.1302/0301-620X.96B3.

[10] D. E. Beverland et al., "HIP ARTHROPLASTY: AVOIDING AND MANAGING PROBLEMS Placement of the acetabular component," vol. 98, no. 1, 2016, doi: 10.1302/0301-620X.98B1. 
[11] W. B. Hiddema, J. F. van der Merwe, and W. van der Merwe, "The Transverse Acetabular Ligament as an Intraoperative Guide to Cup Abduction,” Journal of Arthroplasty, vol. 31, no. 7, pp. 1609-1613, Jul. 2016, doi: 10.1016/j.arth.2016.01.019.

[12] W. van der Merwe, J. van der Merwe, and W. Hiddema, "CAN THE TRANSVERSE ACETABULAR LIGAMENT BE USED AS A LANDMARK FOR INTRA-OPERATIVE ASSESSMENT OF CUP INCLINATION?,” Orthopaedic Proceedings, vol. 96-B, no. SUPP_13, p. 35, Sep. 2014, doi: 10.1302/1358992X.96BSUPP_13.SAOA2013-035.

[13] H. Abe et al., "Is the transverse acetabular ligament a reliable cup orientation guide?," Acta Orthopaedica, vol. 83, no. 5, pp. 474-480, Oct. 2012, doi: 10.3109/17453674.2012.727077.

[14] W. PEDDIE, “Helmholtz's Treatise on Physiological Optics,” Nature, vol. 118, no. 2959, pp. 74-76, 1926, doi: 10.1038/118074a0.

[15] B. Rogers and O. Naumenko, "Perception of straightness and parallelism with minimal distance information," Attention, Perception, and Psychophysics, vol. 78, no. 5, pp. 1381-1391, Jul. 2016, doi: 10.3758/s13414-016-1083-x.

[16] "https://www.corailpinnacle.net/education/additional-materials/tal/surgical-technique." 